\title{
Utilization of Terahertz Spectroscopy for Optical Behavior Determination of Recycled Modified HDPE
}

\author{
Jan Navratil ${ }^{1, a^{*}}$, Miroslav Manas ${ }^{1, b}$, Michal Stanek ${ }^{1, c}$, David Manas ${ }^{1, d}$, Martin \\ Bednarik $^{1, \mathrm{e}}$, Ales Mizera ${ }^{1, f}$ and Tomas Gavenda ${ }^{1, g}$ \\ ${ }^{1}$ Tomas Bata University in Zlin, nam. T.G. Masaryka 5555, 76001 Zlin, Czech Republic \\ aj1navratil@ft.utb.cz (Corresponding author), ’manas@fai.utb.cz, cstanek@ft.utb.cz, \\ ddmanas@ft.utb.cz, 'embednarik@ft.utb.cz, 'fmizera@ft.utb.cz, ggavenda@fai.utb.cz
}

Keywords: Recycling, HDPE, LDPE, Terahertz spectroscopy, Irradiation, Refractive index.

\begin{abstract}
Little research was done to investigate possible utilization of irradiated materials after the end of their lifetime. This research paper deals with the possible utilization of irradiated high-density polyethylene (HDPEx) after its service life. Irradiated HDPE was used as filler into the virgin low-density polyethylene (LDPE). Three material combinations were investigated (powder/powder, granules/powder and granules/grit) and influence of the filler on optical behavior was measured. Terahertz spectroscopy at wide range of frequencies was used for refractive index determination. According to measured data there is significant influence by the amount of the filler. Moreover influence of particle size was also observed. All three combinations have similar curve courses; however the most consistent results were achieved at the powder/powder combination. Behavior of virgin LDPE and virgin HDPE is in correlation with previous findings.
\end{abstract}

\section{Introduction}

Positive effect of irradiation on plastics has been utilized in industry for ages. Discovery of this modification lead to its widespread into many applications e.g. cable insulations, heat-shrinkable products, piping etc. However there are several responses to irradiation from different polymers. The most desired one is radiation crosslinking [1,2].

Crosslinking causes changes in the polymer structure, which results in enhancement of many desired properties. There are two main radiation sources (beta and gamma rays). The most used one in polymer industry is beta rays due to its superior properties $[3,4,5]$.

Irradiated thermoplastics are due to the structural changes losing their natural ability of being remelted. Hence making their reuse impossible [2,5]. This research paper tries to solve this upcoming problem and investigates utilization of irradiated polymer material and its influence on optical properties.

\section{Experiment}

The main goal of this experiment is to determine influence of the amount of filler on refractive index at three material combinations using terahertz spectroscopy.

High-density polyethylene (HDPE). TIPELIN 6300B produced by Slovnaft Petrochemicals, Inc. was used. Products made of this material were originally irradiated by beta radiation with energy 10 $\mathrm{MeV}$ and dose $165 \mathrm{kGy}$. Products were provided after their service life and for purposes of this research was part of them crushed into grit and part of them ground into a powder and used as filler.

Low-density polyethylene (LDPE). Type 780E produced by The Dow Chemical Company was used as a polymer matrix. This material was provided as granules therefore part of them had to be also crushed into a powder.

Measured specimens. Three material combinations were prepared - LDPE granules/HDPEx grit, LDPE granules/HDPEx powder, LDPE powder/HDPEx powder. These raw materials were mixed together in laboratory pneumatic blender in concentrations from 10 to $60 \%$. Thus prepared 
combinations were injection molded in Arburg Allrounder $470 \mathrm{H}$ hybrid injection molding machine. Specimens' shape and dimensions can be seen in Fig. 1.

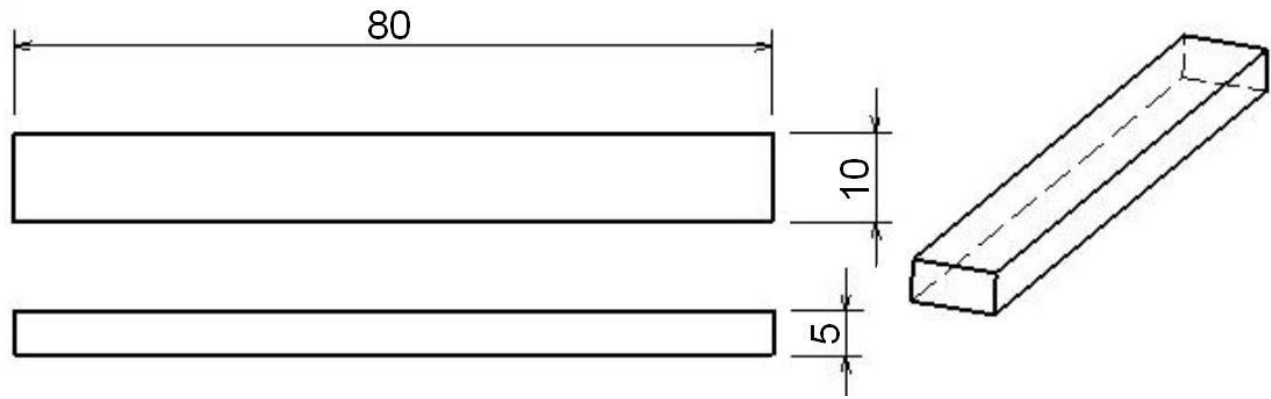

Fig. 1 Specimen shape

Terahertz spectroscopy. TPS ${ }^{\mathrm{TM}}$ Spectra 3000 made by TeraView Ltd. was used for refractive index determination. Measurement was carried out in the vacuum of the machine's working space at ambient temperature $25{ }^{\circ} \mathrm{C}$. Each specimen was measured at 3 different spots, for each 300 scans were performed. Frequency range was from $0.5 \mathrm{THz}$ to $2.5 \mathrm{THz}$. Resulting refractive index was calculated using TPS ${ }^{\mathrm{TM}}$ Spectra 3000 software according to the Eq. 1 where $\mathrm{n}$ is the refractive index, $\mathrm{d}$ is the specimen thickness, $\mathrm{c}$ is the speed of light in vacuum, $\omega$ is the angular frequency of the radiation, $\varphi_{S O}$ and $\varphi_{R O}$ the phases of the specimen and reference terahertz pulses.

$\mathrm{nd}=(\mathrm{c} / \omega)\left(\varphi_{\mathrm{SO}}-\varphi_{\mathrm{RO}}\right)$

\section{Results and discussion}

The only factor to determine optical behavior which was taken into account was refractive index. This factor is one of the most important when designing an optical element.

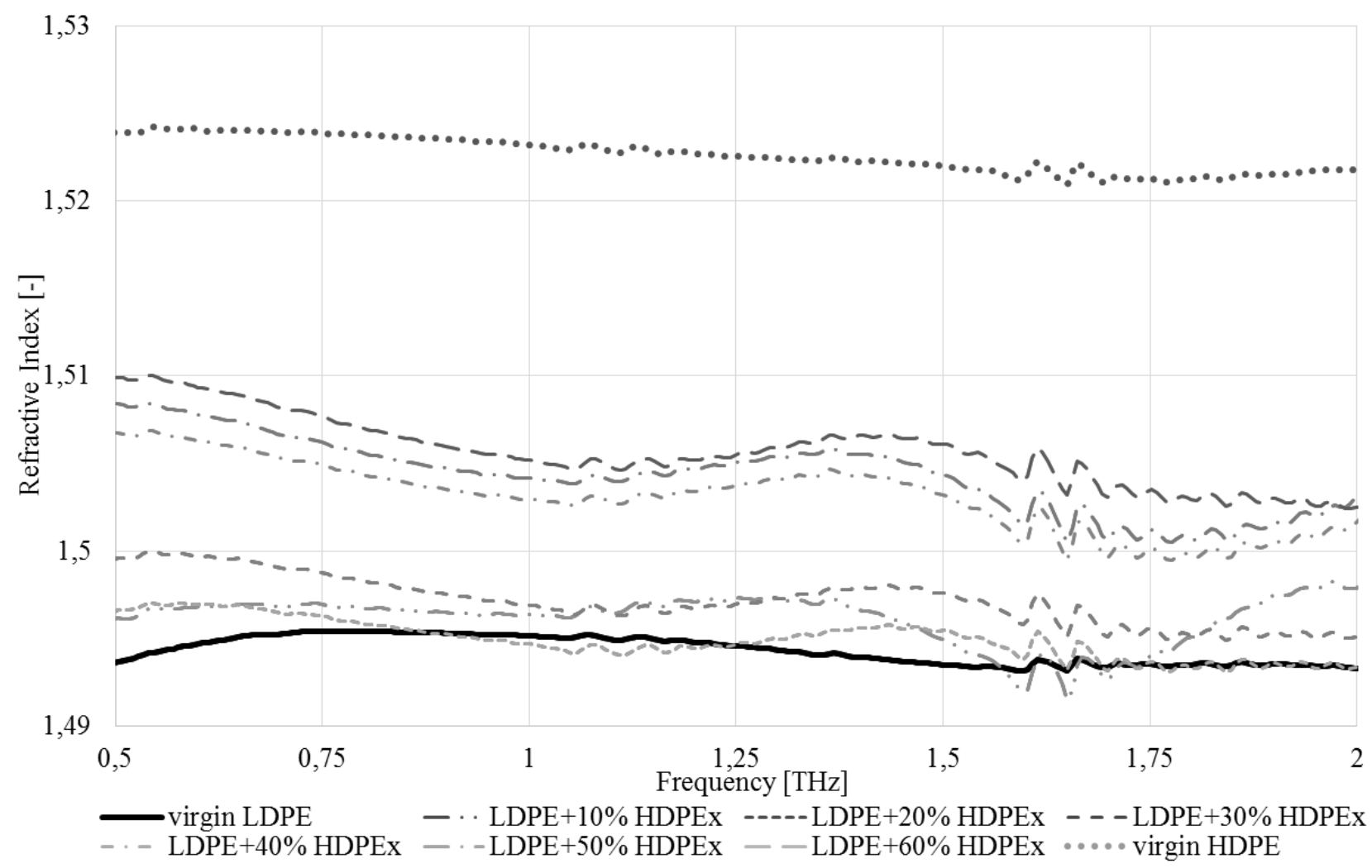

Fig. 2 Refractive index of powder/powder combination

Powder/powder. There were two limiting values measured. Bottom limit represented by refractive index of the virgin LDPE and upper limit represented by refractive index of virgin HDPE as can be seen if Fig. 2. 
Refractive index of the virgin LDPE was around 1.495 and remained almost constant in the whole frequency range. There is only small fluctuation at $1.6 \mathrm{THz}$. Refractive index of the virgin HDPE is slightly decreasing with increasing frequency. It decreased from 1.525 to 1.515 . Fluctuation at 1.6 $\mathrm{THz}$ remained unchanged. All these findings are in correlation with already known optical behavior of the virgin polymers [6]. Course of curves of virgin LDPE filled with recycled HDPEx is on the other hand much more frequency dependent, which might suggest some instabilities in their structure. There is also increase of refractive index with increasing amount of the filling, where the highest value of the refractive index was observed at the highest concentration of filling and vice versa.

Granules/powder. As is depicted in Fig. 3 virgin LDPE specimens produced from granules have higher refractive index than those produced from powder. Decrease of refractive index with increasing frequency is also more significant. It decreased from 1.513 to 1.508 . Course of the curve of the virgin HDPE is the same as in Fig. 2. Values of the refractive index of granules/powder compounds varies similarly to powder/powder compounds. However unlike powder/powder combination the highest value of refractive index was not measured at the highest concentration of filling. The highest refractive index of filled LDPEs was measured at $50 \%$ concentration. This may be caused by problematic homogenization during processing. This can also be seen from course of its curve which significantly deviates from course of other curves.

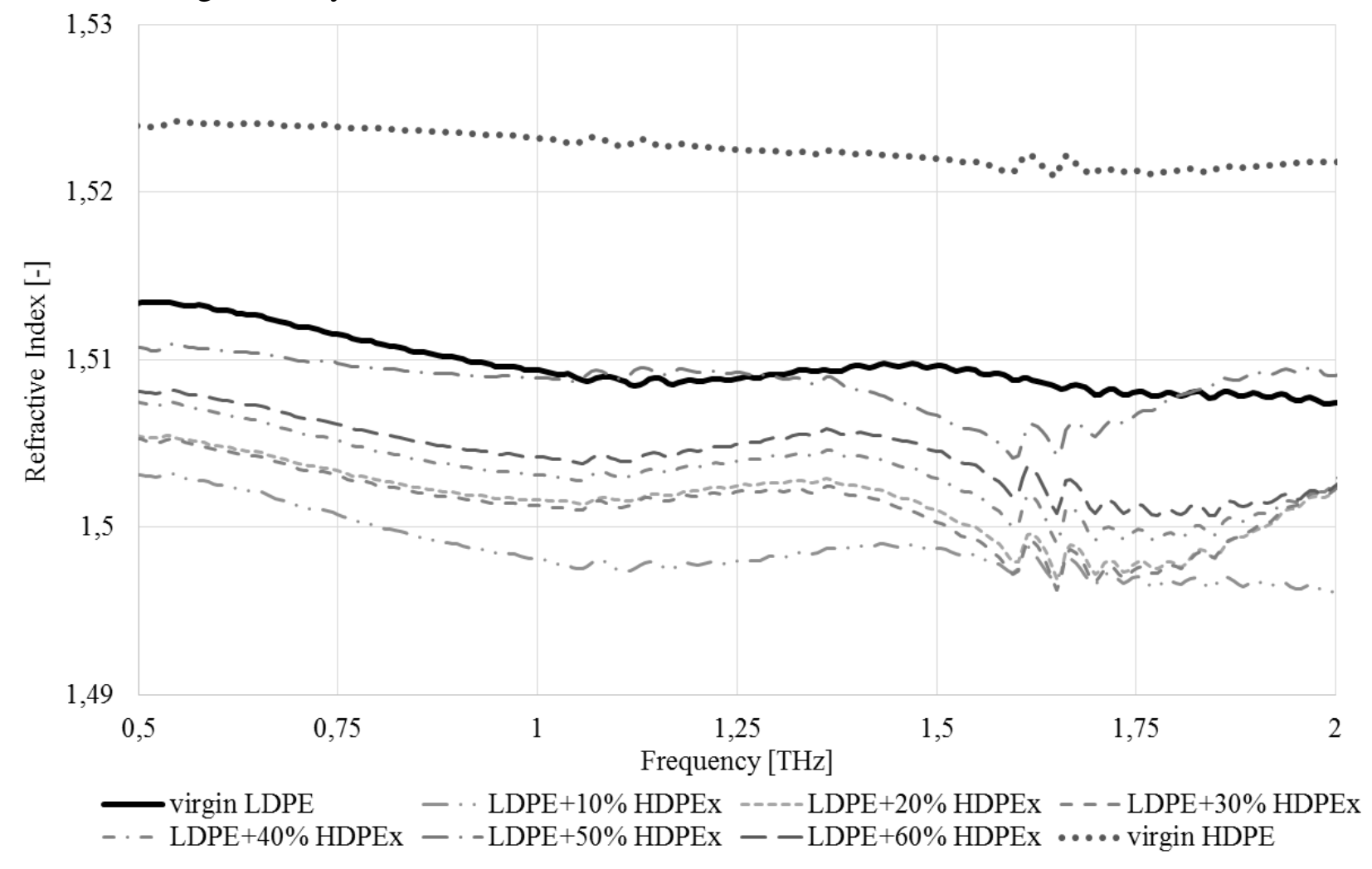

Fig. 3 Refractive index of granules/powder combination

Granules/grit. Last material combination can be seen in Fig. 4. Values of refractive index of virgin LDPE and virgin HDPE are the same as in Fig. 3. All concentrations of LDPE filled with the grit of recycled HDPEx show the most unpredictable behavior. Curves courses are fully comparable with the previous ones but values of individual refractive indexes greatly vary. The lowest value was achieved at $60 \%$ concentration and the highest values was achieved at $40 \%$ concentration. The reason of this unpredictable behavior is probably much bigger particle size of the filler (ten times bigger than in case of powder) which results in this problematic quantification. 


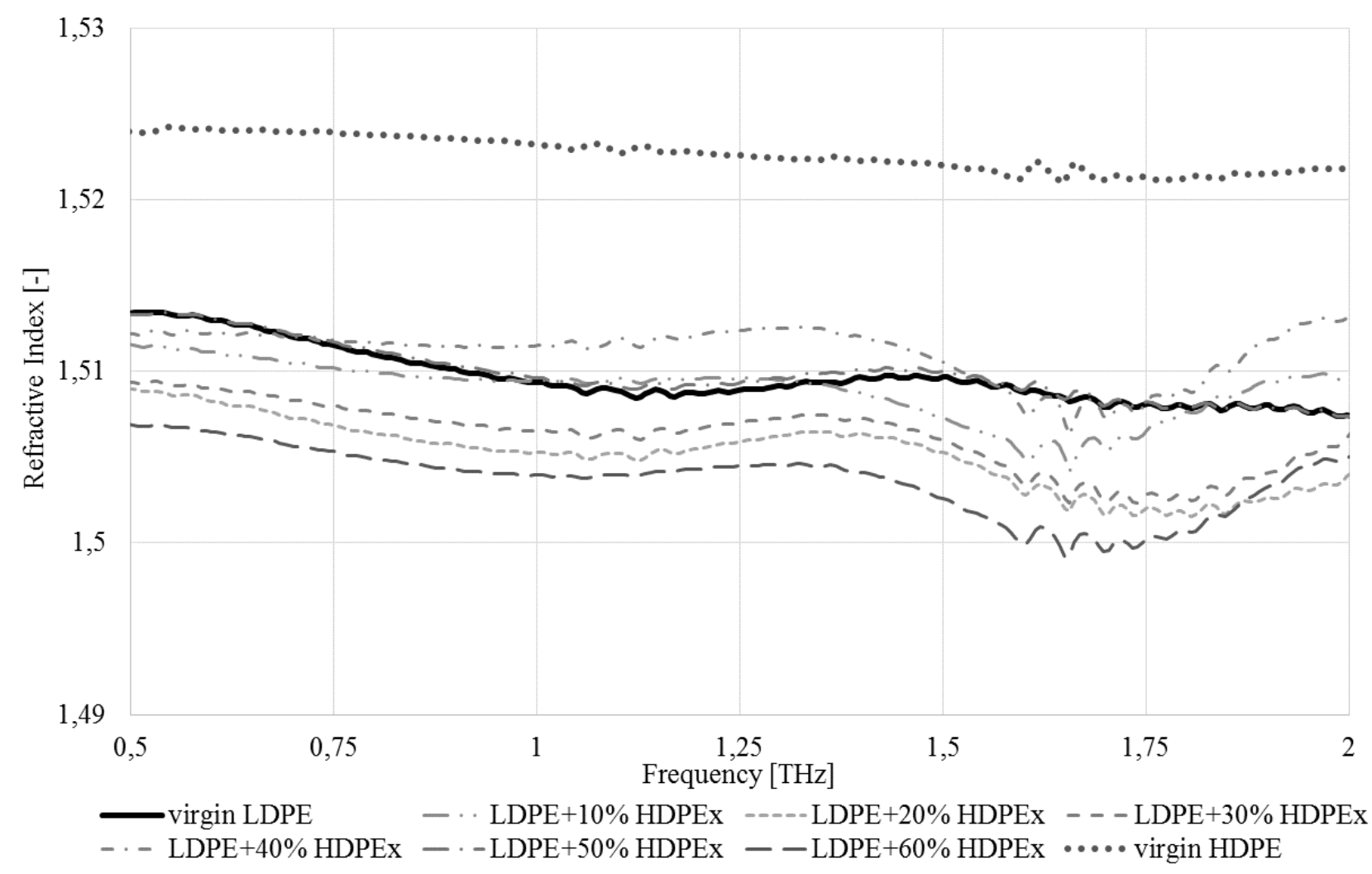

Fig. 4 Refractive index of granules/grit combination

\section{Summary}

This research paper investigates optical behavior of recycled modified polymer material used as filler. Recycled irradiated HDPE was used as filler into the virgin LDPE. Optical behavior was represented by refractive index measured by terahertz spectroscopy. Measurement was carried out at a wide range of frequencies to determine possible usage of these materials in optics. Results show that there is a significant influence of particles size. Powder/powder combination showed the most consistent course of refractive index whereas other combinations results were more prone to some deviations. Therefore powder/powder combination is the best combination in terms of optics.

\section{Acknowledgment}

This paper is supported by the internal grant of TBU in Zlin No. IGA/FT/2014/016 funded from the resources of specific university research and by the European Regional Development Fund under the project CEBIA-Tech No. CZ.1.05/2.1.00/03.0089.

\section{References}

[1] R.L. Clough and S.W. Shalaby: Irradiation of Polymers: Fundamentals and Technological Applications, American Chemical Society, (1996).

[2] E. Adem et al.: Radiat. Phys. Chem., Vol. 52, No. 1-6 (1998), p. 171.

[3] J. Gehring: Radiat. Phys. Chem., Vol. 57, No. 3-6 (2000), p. 361.

[4] D.W. Clegg and A.A. Collyer: Irradiation Effect on Polymers, Elsevier Science Publications, (1991).

[5] A. Bhattacharya: Prog. Polym. Sci., Vol. 25, No. 3 (2000), p. 371.

[6] S. Wietzke et al.: J. Mol. Struct., Vol. 1006, No. 1-3 (2011), p. 41. 